\title{
NERVUS TERMINALIS IN DOGFISH (SQUALUS ACANTHIAS, ELASMOBRANCHII) CARRIES TONIC EFFERENT IMPULSES
}

\author{
THEODORE H. BULLOCK ${ }^{1}$ and R. GLENN NORTHCUTT ${ }^{2}$ \\ ${ }^{1}$ Neurobiology Unit, Scripps Institution of Oceanography, and Department of Neurosciences, University \\ of California, San Diego, La Jolla, CA 92093, and 'Division of Biological Sciences, University of \\ Michigan, Ann Arbor, MI 48109 and Friday Harbor Laboratories, University of Washington, Friday \\ Harbor, WA 98250 (U.S.A.)
}

(Received August 17th, 1983; Revised version received October 10th, 1983; Accepted November 22nd, 1983)

Key words: cranial nerve - chemosensory nerve - pheromone receptor - centrifugal output in sensory nerve

Recordings from the intact nervus terminalis with a hook electrode or from a stump of the divided nerve with a suction electrode show a tonic, irregular discharge of broad, low frequency spikes in ca. 4-6 units. These nerve impulses are efferent from the brain. The mean frequency of discharge is not influenced by various chemical, thermal, tactile, acoustic, photic, vibratory and electric field stimuli but is decreased by certain forms of mechanical stimuli, presumably acting on the lateral line organs of the lateral aspect of the head. We have not succeeded in recording from afferents. The nerve consists of $>1000$ unmyelinated axons, mostly $<1 \mu \mathrm{m}$, a very few $>1.5 \mu \mathrm{m}$ in diameter; presumably the efferents recorded from were these larger fibers.

A cranial nerve distinct from, and morphologically rostral to, the olfactory nerve was illustrated by Fritsch [2] in 1878 from the brain of the dogfish, Mustelus. Pinkus [10] was the first to describe the course of the nerve. The commonly used name, nervus terminalis, was proposed by Locy $[6,7]$ based on Squalus and a number of other elasmobranch fishes. All descriptions agree that the nerve is ganglionic and that it runs close to or with the olfactory tract and bulb. Sometimes there are two ganglia along the nervus terminalis. The ganglion or ganglia are variously situated, either beside the olfactory tract or bulb, or buried, or even dispersed as scattered cells within them. Except for birds, this cranial nerve and ganglion are now known in some members of each of the classes of jawed vertebrates, including among many mammals, the human, though it has not been identified in all the species examined in each class $[4,8]$. There are significant differences between species, best known among teleost $[3,12]$.

In elasmobranchs such as Squalus the nerve is distinct from the olfactory tract, although in contact with it in a common connective tissue. The ganglion may be along the course of the nerve or at its rostral end, beside the olfactory tract or bulb. 
The peripheral termination of the sensory axons of nervus terminalis is said by the older authors to be in the olfactory epithelium. Several authors $[5,12]$ have suggested that the nerve is both sensory - from the same epithelium as the olfactory nerve - and also visceral motor - on the same plan as the autonomic nervous system. Rossi and co-workers $[11,12]$ give evidence that this mixed nature is true for some teleosts (Scorpaena) whereas others (Tinca) have purely afferent nerves.

A recent revival of interest in this nerve has resulted in new anatomical findings and some functional speculations. The nervus terminalis proves to be part of a remarkable system [1]. Horseradish peroxidase injection into the eye has revealed labeled cells in the terminal nerve ganglion (also called the nucleus olfactoretinalis in some teleosts), indicating the direct projection of their axons to the retina; the nerve also projects to several nuclei in the forebrain. Preliminary evidence obtained by one of us (R.G.N.) from Raja points to the same conclusion for elasmobranchs. Munz et al. [9], Springer [13] and Stell et al. [14] found that the axons as they course through the nervus terminalis and the brain, as well as the cells in the ganglion, contain luteinizing hormone-releasing hormone (LH-RH). Visualizing the fibers with immunofluorescence techniques confirms the projections to retina and forebrain targets. Demski and Northcutt [1] give reasons to believe that the functions of the nervus terminalis system may include chemoreception for pheromones.

The purpose of the present study was to attempt to record electrical activity from the nervus terminalis, as a step in elucidating its function. We were able to record nerve impulses, which proved to be efferent; we have not succeeded in recording any afferent activity, either spontaneous or elicited by various sensory stimuli.

Spiny dogfish (Squalus acanthias) of 1-1.5 kg were anesthetized with MS222 (tricaine methane sulfonate) and a surgical exposure was made uncovering the rostral end of the telencephalon, the olfactory tracts and the caudal portion of the olfactory bulbs, from the dorsal side. Injecting D-tubocurarine, $0.4-0.6 \mathrm{mg} / \mathrm{kg}$ intramuscularly or intravenously, reduced the vigor of the occasional spontaneous swimming movements so that a head holder together with rubber bands supporting the body, via small fish hooks inserted in the middorsal line, provided sufficient immobilization. A stream of sea water was led into the mouth at a rate which caused a gentle flow from most of the gill slits.

The nervus terminalis (Fig. 1A) was carefully freed from its medial position on the olfactory tract and followed proximally across the rostral pole of the cerebral hemisphere, about halfway toward its disappearance in the midsagittal fissure. By blunt dissection, freeing the nerve distad from that point to the level of the olfactory bulb, at least $7 \mathrm{~mm}$ of slack nerve is available. This permits lifting the nerve with one or two hook electrodes into the air, preserving both its proximal and its distal connections. In a later phase of the experiment, after cutting the nerve, either of the stumps was drawn into a close fitting suction electrode, permitting many hours of continuous recording. The forms of stimuli used are described below.

A continuous background activity is seen in the nerve lifted into air on a hook 


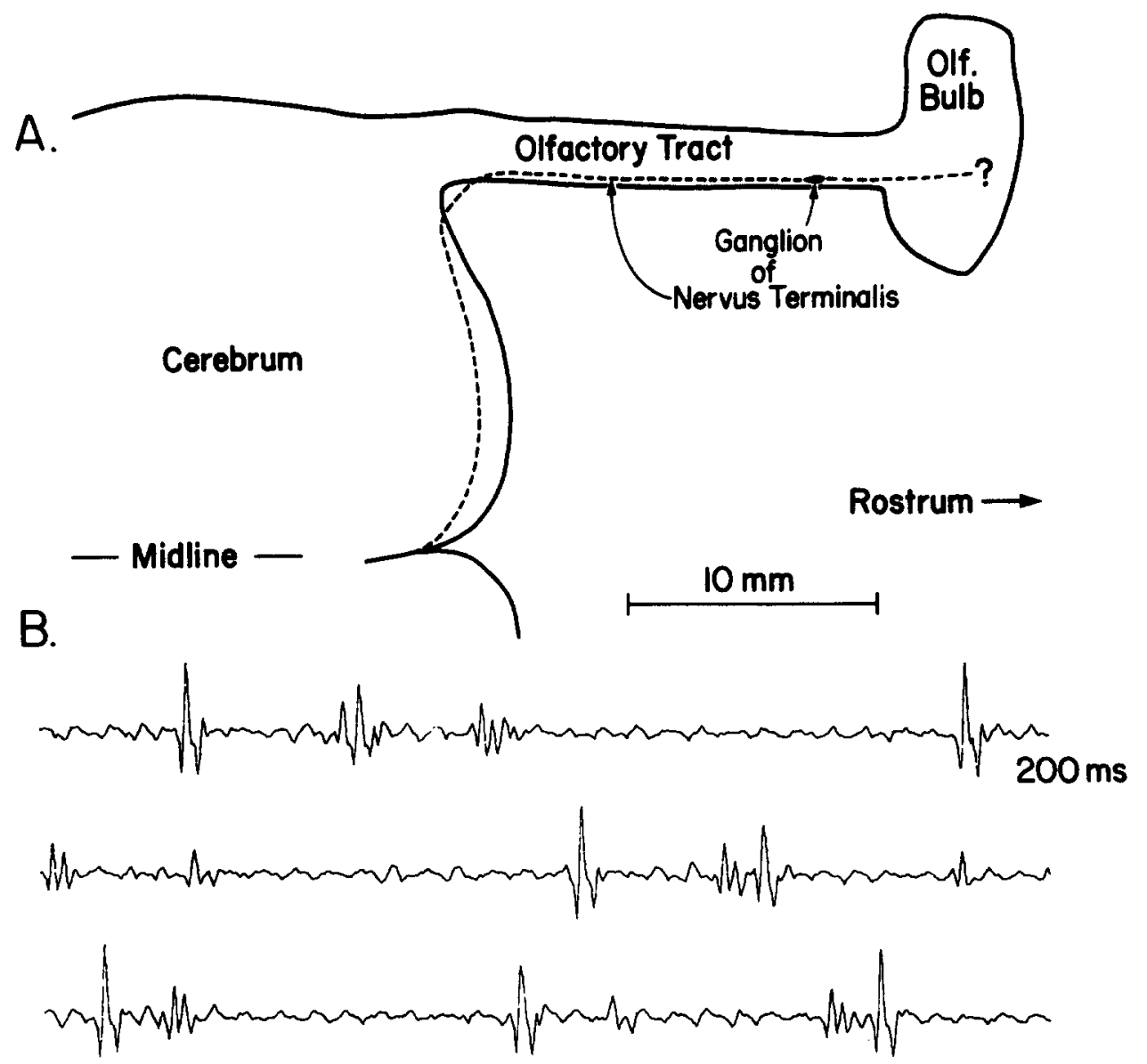

Fig. 1. A: diagrammatic dorsal view of the rostral end of the telencephalon, olfactory tract and bulb to show the nervus terminalis and its ganglion. The proximal end of the nerve enters the brain in the sagittal fissure, via the anterior neuroporic recess. B: tonic spike activity recorded in the proximal portion of the whole nervus terminalis lifted into the air on a hook electrode; random samples of single sweeps, each $200 \mathrm{~ms}$ long; amplifier filters at $100-1000 \mathrm{~Hz}$; note several repeated waveforms.

electrode (Fig. 1B). This activity consists of unit spikes of approximately 3-5 shapes firing altogether about 5-10 spikes per second. All the spike shapes have a rather long duration, ca. 4-6 ms.

The possibility that these spikes are muscle action potentials can be discounted on the following grounds. The nearest muscle is more than a centimeter away and no muscle action potentials are recorded when the electrode is placed at various points on the cartilage or brain. The observed spikes are unaffected by the position of the reference electrode, which may be close to the active electrode, in the pool of cerebrospinal fluid or elasmobranch saline solution or on the nerve itself. The spikes are seen only when the nerve is lifted out of the pool or when the proximal 
(caudal) stump is drawn into a close fitting suction electrode under the water surface.

A variety of forms of stimulation fails to accelerate or decelerate the tonic discharge of these spikes. We tried water extracts of tissue of a plaice and washings of the oviduct of ratfish (Hydrolagus colliei), dilutions of mucus from dogfish skin, dilutions of dogfish bile and of cloacal washings, water extracts of dogfish liver, sea water in which a crab had been crushed, and solutions of aspartate, glutamate, proline, alanine and glycine (each $10^{-3} \mathrm{M}$ in sea water). A small jet of sea water or of distilled water into the olfactory sac, whether slow or fast, is ineffective. Also ineffective in altering the tonic discharge are the following: touching the skin near the opening of the olfactory sac, scratching the skin with a broken glass pipette all over the body, tapping on the tank or head holder, voices and various artificial sounds, shadows, visible movement and flashing light into either eye, and the make or break of an electric field adequate to excite ampullae of Lorenzini strongly.

The tonic discharge of spikes in the nervus terminalis is unaffected by local procaine applied to the distal end of the freed segment where it rejoins the olfactory bulb, or by crushing the nerve distally or in the segment in air, or by cutting the nerve and recording from the proximal stump. The spikes are abolished by procaine applied proximally, i.e. to the point where the nerve joins the telencephalon, or by crushing near the telencephalon. After dividing the nerve, no spikes are seen in the distal stump drawn into a suction electrode either tonically or after stimulation with the various agents listed above. From these facts it is clear that the spikes are centrifugal, i.e. efferent from the brain toward the distal end of the nerve or its ganglion.

The mean level of about 5-10 centrifugal spikes per second is typically maintained for many hours although the intervals are irregular; fluctuations of the mean are not conspicuous. As seldom as once in tens of minutes there may be a slow down to $2 / \mathrm{s}$ for a few seconds and very rarely there is a spontaneous silence for $3-5$ seconds.

A few forms of stimulation can alter the tonic discharge of centrifugal spikes. Gently swishing water near the lateral aspect of the head on the same side, for example by a single pass of a $7 \mathrm{~mm}$ glass rod parallel to the side of the head, $2 \mathrm{~cm}$ away and moving $10 \mathrm{~cm}$ in $0.5 \mathrm{~s}$, can cause a slowing or complete suppression of spike frequency, after a latency of up to $4 \mathrm{~s}$, lasting for $5 \mathrm{~s}$ or more, followed by a slow return of the tonic discharge in 5-10 s, sometimes with a few seconds of rebound hyperactivity. Local jets of water in the same area are ineffective and swishing over large areas elsewhere on the body is ineffective. A single weak bump of the head holder or a single gentle puff of air on the water surface near the head can cause the suppression of spikes.

In order to evaluate the finding of a few active units and the failure to record from what we presume to be the majority of the fibers in the nerve we fixed some material for electron microscopy. The freshly exposed nerve was flooded with a mixture of $2.5 \%$ glutaraldehyde and $2 \%$ paraformaldehyde in phosphate buffer; the nerve was 


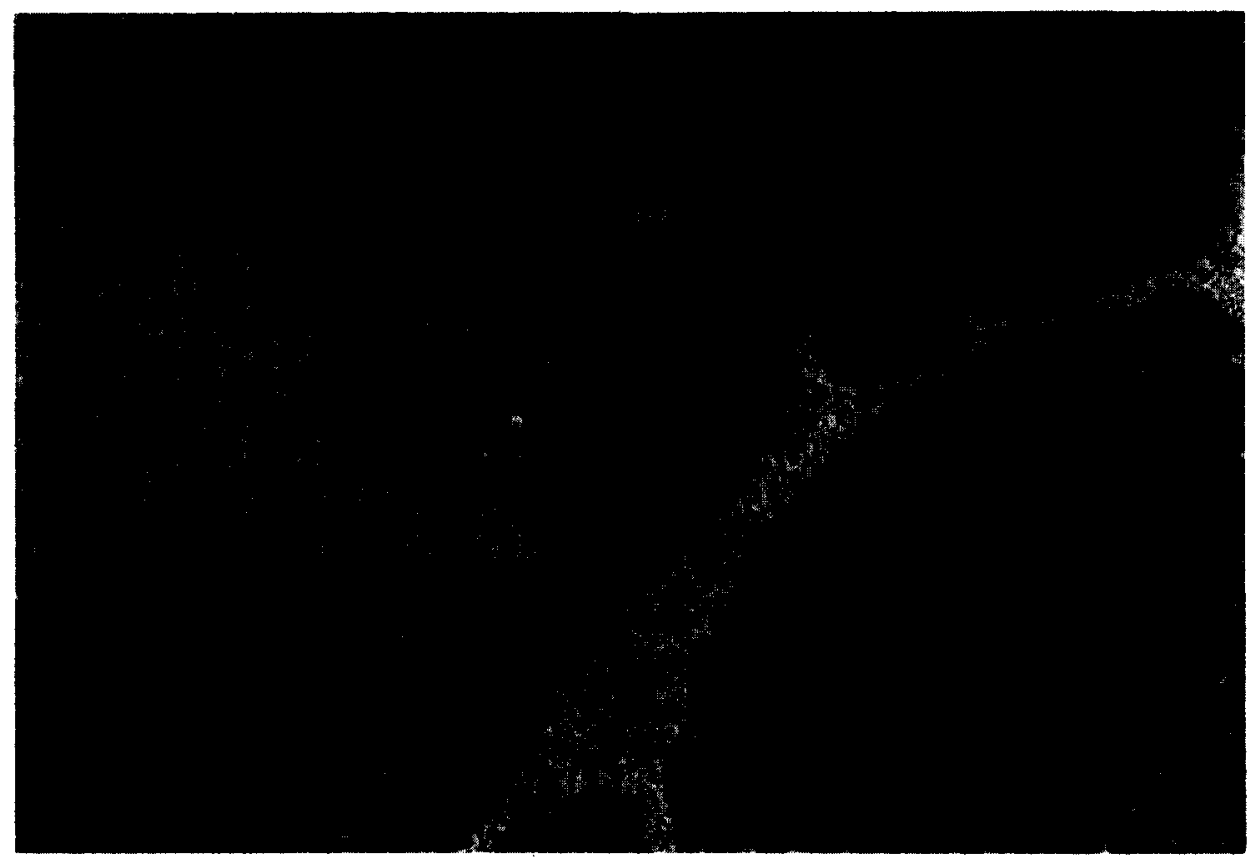

Fig. 2. Electron micrograph of the nervous terminalis in transverse section. Bar scale $=1 \mu \mathrm{m}$. Courtesy of R.D. Fields.

dissected from the olfactory tract immediately and immersed in the fixative for 1 $\mathrm{h}$, then postfixed in $1.5 \%$ osmic acid. R.D. Fields kindly cut sections and stained them with uranyl acetate and lead citrate and prepared electron micrographs. The nerve consists of more than 1000 fibers, all unmyelinated (Fig. 2), with widely varying diameters ranging from much less than $1 \mu \mathrm{m}$ to something more than $2 \mu \mathrm{m}$. Most fibers are $<0.6 \mu \mathrm{m}$; a very few are $>1.5 \mu \mathrm{m}$ in diameter. Many axons, especially the largest ones, contain more or less dense granules, often surrounded by a vesicular wall; the larger granules reach $0.2 \mu \mathrm{m}$. Glial nuclei and processes are conspicuous but the processes generally envelop large bundles of naked axons.

The main significance of these results is perhaps to call attention to a tractable preparation for studying the physiology of a little known cranial nerve. The nervus terminalis has not previously been studied physiologically but its anatomical relations have suggested some interesting functions, including chemoreception of pheromones and direct modulation of the retina. Rossi et al. [12] had already concluded on anatomical grounds that the nerve is not only sensory but also motor, as others, including Larsell [5], had done earlier, presumably supplying glandular, vascular or other effectors in the mucosa.

Our finding of efferent activity raises the question whether this represents centrifugal control of receptors, either of the nervus terminalis or of the olfactory nerves, or visceral efferent control of some peripheral effector. We cannot at present suggest more specific meanings of the efferent impulses observed since the targets of this output are not known. Even the distal (rostral) terminations of the 
nervus terminalis as a whole are not agreed upon. Both olfactory epithelium and olfactory bulb have been implicated. If they include sensory endings in the epithelium, it will be important to determine whether the efferent fibers go to those endings. Other chemoreceptors such as the olfactory and gustatory are not known to receive efferent innervation. The ganglion of the nervus terminalis in elasmobranchs should be examined with respect to the presumed synapses. Rossi et al. [12], in the first electron microscope study of this nerve and ganglion, report some indications in teleost of synapses in the ganglion, due to terminals of myelinated axons on one of two classes of cells in the ganglion. There appear to be no myelinated fibers in the nerve in Squalus, but the efferents are probably the largest fibers, which often carry large, dense granules.

There is no indication of the origin of the centrifugal discharge but experimental anatomical methods that exploit retrograde transport of markers should be applied to the proximal stump of the divided nerve. The suppression of efferent discharge by water movement stimuli to the facial region points to a connection of the centrifugal neurons with one of the projections of the lateral line mechanoreceptors.

Since we only used gross electrodes, the failure to record afferent activity in the fine unmyelinated fibers is not surprising and there is reason to hope that microelectrodes will succeed in recording from afferent units in the nerve or from cell bodies in its ganglion, permitting direct search for the adequate stimuli.

Aided by grants to T.H.B. and to R.G.N. from the National Science Foundation and the National Institutes of Health.

1 Demski, L.S. and Northcutt, R.G., The terminal nerve: a new chemosensory system in vertebrates? Science, 220 (1983) 435-437.

2 Fritsch, G., Untersuchungen über den feineren Bau des Fischgehirns, Berlin, 1878, pp. 1-94.

3 Grüneberg, H., A ganglion probably belonging to the $N$. terminalis system in the nasal mucosa of the mouse, Z. Anat. Entwickl.-Gesch., 140 (1973) 39-52.

4 Johnston, J.B., Nervus terminalis in reptiles and mammals, J. comp. Neurol., 23 (1913) 97-120.

5 Larsell, O., Studies on the nervus terminalis: mammals, J. comp. Neurol., 30 (1918) 3-68.

6 Locy, W.A., A new cranial nerve in selachians. In G.H. Parker (Ed.), Mark Anniversary Volume, Henry Holt, NY, 1903, pp. 39-55.

7 Locy, W.A., On a newly recognized nerve connected with the forebrain of selachians, Anat. Anz., 26 (1905) 33-63 and 111-123.

8 McKibben, P.S., The nervus terminalis in urodele Amphibia, J. comp. Neurol., 21 (1911) 261-309.

9 Munz, H., Class, B., Stumpf, W.E. and Jennes, L., Centrifugal innervation of the retina by luteinizing hormone releasing hormone (LHRH)-immunoreactive telencephalic neurons in teleostean fishes, Cell Tiss. Res., 222 (1982) 313-323.

10 Pinkus, F., Über einen noch nicht beschriebenen Hirnnerven des Protopterus annectens, Anat. Anz., 9 (1984) 562-566.

11 Rossi, A. and Basile, A., Comparative study of the topography of the ganglion cells of the terminal nerve of teleosts, Atti Accad. Naz. Lincei, Rend. Cl. Sci. Fis. Mat. Natur., 45 (1968) 635-642.

12 Rossi, A., Basile A. and Palombi, F., Speculations on the function of the nervus terminalis system in teleosts, Riv. Biol. (Perugia), 65 (1973) 401-409.

13 Springer, A., Retinopetal cells in the goldfish olfactory bulb, Invest. Ophthalmol. Vis. Sci., 22, Suppl. (1982) 246.

14 Stell, W.K., Walker, S.E., Chohan, K.S. and Ball, A.K., The goldfish nervus terminalis:' a luteinizing hormone-releasing hormone and molluscan cardioexcitatory peptide immunoreactive olfactoretinal nathwav Pror nat Arad Sri IISA in nrese 